\title{
An Efficient Approach to Discovering Sequential Patterns in Large Databases
}

\author{
Show-Jane Yen and Chung-Wen Cho \\ Department of Computer Science and Information Engineering \\ Fu Jen Catholic University, Taipei 242, Taiwan, ROC \\ \{sjyen, Cho\}@csie.fju.edu.tw
}

\begin{abstract}
Mining sequential patterns is to discover sequential purchasing behaviors of most customers from a large amount of customer transactions. The previous approaches for mining sequential patterns need to repeatedly scan the large database, and take a large amount of computation time to find frequent sequences, which are very time consuming. In this paper, we present an algorithm SSLP to find sequential patterns, which can significantly reduce the number of the database scans. The experimental results show that our algorithms are more efficient than the other algorithms.
\end{abstract}

\section{Introduction}

Because the capacity of the storage is getting larger, large amount of data can be stored in the database. Potential useful information may be embedded in the large databases. Hence, how to discover the useful information exists in such databases is becoming the popular field in the computer science. The purpose of data mining [1], [3], [4], [5] is to discover the useful information from the large databases, such that the quality of decision making can be improved.

A transaction database consists of a set of transactions. A transaction typically consists of the transaction identifier, the customer identifier (the buyer), the transaction date (or transaction time), and the items purchased in this transaction. Mining sequential patterns [1], [2], [6] is to find the sequential purchasing behavior of most customers from a large transaction database. For example, there is a sequential pattern $<\{$ TERMINATOR, TERMINATOR 2$\}\{$ TRUE AND FALSE $\}\{J U N I O R\}>70 \%$ is discovered from the transaction database in a video rental store, which means that seventy percent of the customers rent TRUE AND FALSE after renting both TERMINATOR and TERMNATOR 2, and then they rent JUNIOR after enjoying TRUE $A N D$ FALSE. The manager can use this information to recommend the new customers to rent TRUE AND FALSE and JUNIOR when they rent TERMINATOR and TERMINATOR 2.

The definitions about mining sequential patterns are presented as follows: An itemset is a non-empty set of items. An itemset $X$ is contained in a transaction $T$, if $X \subseteq T$, and $X$ is a sub-itemset of $T$. 
A sequence is an ordered list of the itemsets. A sequence $s$ is denoted as $\left\langle s_{1}, s_{2}, \ldots\right.$, $\left.s_{n}\right\rangle$, where $s_{i}$ is an itemset. A sequence $\left\langle a_{1}, a_{2}, \ldots, a_{n}\right\rangle$ is contained in another sequence $\left\langle b_{1}, b_{2}, \ldots, b_{m}>\right.$ if there exist integers $i_{1}<i_{2}<\ldots<i_{n}, 1_{i} \varnothing i_{k}$ iØm such that $a_{1} \subseteq b i_{1}, \ldots, a_{n} \subseteq b i_{n}$, then $\left\langle a_{1}, a_{2}, \ldots, a_{n}>\right.$ is a subsequence of sequence $<b_{1}, b_{2}, \ldots$, $b_{m}>$. A maximum sequence is a sequence that is not contained in any other sequence.

A customer sequence is the list of all the transactions of a customer, which is ordered by increasing transaction-time. A customer sequence $c$ supports a sequence $s$ if $s$ is contained in $c$. The support for a sequence $s$ is the number of customer sequences that supports $s$. If the support for a sequence $s$ satisfies the user-specified minimum support threshold, then $s$ is called frequent sequence. Otherwise, $s$ is a non-frequent sequence. The length of a sequence $s$ is the number of itemsets in the sequence. A sequence of length $k$ is called a $k$-sequence, and a frequent sequence of length $k$ a frequent $k$-sequence. In general, before generating the frequent sequences, we need to generate the candidate sequences, and scan the database to count the support for each candidate sequenc to decide if it is a frequent sequence. A candidate sequence of length $k$ is called a candidate $k$-sequence.

For the previous approaches [1], [2] they need to generate a large number of candidates to be counted and make multiple passes over the large database, which are very time consuming. In this paper, we present an algorithm SSLP to discover all the sequential patterns. This algorithm can reduce the number of database scans. Moreover, it can effectively decrease the number of candidates to be counted.

The rest of this paper is organized as follows: Section 2 presents the algorithm to discover sequential patterns. The performance evaluation and the experimental results are shown in section 3 .

\section{Mining Sequential Patterns}

We refer to [1] to decompose our algorithms into the same five phases. However, The major work of mining sequential patterns is to find all the frequent sequences. Hence, in the Sequence Phase, we present an efficient algorithm SSLP to generate all the frequent sequences from a transformed transaction database.

\subsection{Algorithm SSLP (Segmental Smallest and Largest Position)}

Let $L_{k}$ be the set of the $k$-frequent sequences, and $C_{k}$ be the set of the candidate $k$ sequences.

Definition 1. Let a sequence $\mathrm{s}=\left\langle\mathrm{s}_{1}, \mathrm{~s}_{2}, \ldots, \mathrm{s}_{\mathrm{n}}\right\rangle$, and a customer sequence $\mathrm{c}=\left\langle\mathrm{c}_{1}\right.$, $\left.\mathrm{c}_{2}, \ldots, \mathrm{c}_{\mathrm{m}}\right\rangle$. If there exists $\mathrm{i}_{1}<\mathrm{i}_{2}<\ldots<\mathrm{i}_{\mathrm{n}} j A 1 ; \emptyset \mathrm{i}_{\mathrm{k}} \not \emptyset m$, such that $\mathrm{s}_{1} \subseteq \mathrm{ci}_{1}, \ldots, \mathrm{s}_{\mathrm{n}} \subseteq \mathrm{ci}_{\mathrm{n}}$, then the position of $\mathrm{s}$ in $\mathrm{c}$ is $\mathrm{i}_{\mathrm{n}}$. Otherwise, the position is 0 .

For example, consider the customer sequence 3 in Table 1 , the position of 2 sequence $<\{\mathrm{A}\}\{\mathrm{B}\}>$ is 3 . 
Definition 2. The position pair of a sequence s corresponding to a customer sequence $c$ is denoted as $(\mathrm{F}, \mathrm{L})$, where $\mathrm{F}$ and $\mathrm{L}$ are the minimum value and the maximum value among all the positions of $\mathrm{s}$ in $\mathrm{c}$, respectively. If $\mathrm{s}$ is not contained in $\mathrm{c}$, then the position pair of $\mathrm{s}$ in $\mathrm{c}$ is $(0,0)$. If the position of $\mathrm{s}$ in $\mathrm{c}$ is $\mathrm{p}$, then the position pair of $\mathrm{s}$ is $(\mathrm{p}, \mathrm{p})$, where $1_{i} \emptyset \mathrm{p} ; \emptyset$ the number of itemsets in $\mathrm{c}$.

For example, consider the customer sequence 3 in Table 1, the position pair of 2sequence $\langle\{\mathrm{A}\}\{\mathrm{B}\}>$ is $(3,3)$.

Table 1. The transformed transaction database $(D B)$

\begin{tabular}{ll}
\hline CID & Transformed customer sequence \\
\hline 1 & $<\{$ A,B,D $\{\{\mathrm{A}\}\{\mathrm{B}\}>$ \\
2 & $<\{\mathrm{A}\}\{\mathrm{B}\}\{\mathrm{A}, \mathrm{B}, \mathrm{C}, \mathrm{D}\}>$ \\
3 & $<\{\mathrm{A}, \mathrm{B}, \mathrm{D}\}\{\mathrm{A}\}\{\mathrm{B}\}\{\mathrm{C}\}>$ \\
4 & $<\{\mathrm{A}, \mathrm{C}\}\{\mathrm{B}, \mathrm{C}\}\{\mathrm{C}\}>$ \\
\hline
\end{tabular}

SSLP partitions $D B$ into some segments. For each segment, SSLP only needs to record the position pair of each candidate sequence corresponding to each customer sequence in the segment. A segment consists of $n$ customer sequences, where $n$ is the length of the segment. The i-segment support for a sequence $s$ is the number of the customer sequences which support $s$ in the $i^{\text {th }}$ segment. SSLP scans the database pass by pass. For the $(k / 2)^{\text {th }}(\mathrm{k}=2,4, \ldots)$ pass, SSLP scans $D B$ from the first segment to the $m^{\text {th }}$ segment, and generates $L_{k}$ and $L_{k-1}$. For the $i^{\text {th }}$ segment scan, SSLP computes the $i$ segment support for each candidate sequence.

Assume that $S S L P$ has scanned the $i^{\text {th }}$ segment. The current support $\mathrm{CS} s(i)$ of a sequence $s=1$-segment support of $s+\ldots+i$-segment support of $s$. The maximum support of $s$ is a value that the support of $s$ is never greater than this value. Let the maximum support of $a=\left\langle a_{1}, \ldots, a_{k}\right\rangle$ be denoted as maxSa $(i)$, and the $i$-segment support of 1 -sequence $a_{j}$ be $S_{i j}$. The initial maximum support max $S a(0)$ of $a$ is defined as :

$\max _{a}(0)=\sum_{i=1}^{i=m}\left\{S i_{1}, \ldots, S i_{k}\right\}, m$ is the number of the segments in the database

After scanning the $i^{\text {th }}$ segment, the maximum support of the $k$-sequence $a$ can be obtained by the following expression :

$$
\operatorname{maxS}_{a}(i)=\operatorname{maxS}_{a}(i-1)_{i} Đ\left(\min \left\{S i_{1}, \ldots, S i_{k}\right\}_{i} Đ i \text {-segment support of } a\right)
$$

For example, the transaction database $D B$ (Table 1) is partitioned into 2 segments, and the minimum support is set to 3 . Hence, the customer sequences 1 and 2 are in the first segment, and the customer sequences 3 and 4 are in the second segment. The 1segment supports and 2-segment supports of all the frequent 1-sequences can be found in the Transformation Phase, which are shown in Table 2. In the following, we use this example throughout this section and describe the three steps for the $(1 / 2) k$ pass $(k=2,4, \ldots)$ for $S S L P$. 
Step 1. Generate $C_{k}$ from $L_{k-1}$, compute the initial maximum support of each candidate $k$-sequence, and then prune the candidate $k$-sequences that cannot be the frequent $k$ sequences from $C_{k}$.

We refer to [1] to generate $C_{k}$ form $L_{k-1}$. After generating candidate $k$-sequences $C_{k}$, if $C_{k}$ is not empty, then the candidates in $C_{k}$ need to be further pruned. Otherwise, $S S L P$ terminates. If one of the $(k-1)$-subsequences of the candidate $k$-sequence $s$ is not in $L_{k-1}$, then s is pruned from $C_{k}$.

Table 2. The segments supports of frequent 1-sequences

\begin{tabular}{c|cc|c|cc}
\hline \multirow{2}{*}{$\begin{array}{c}\text { Frequent 1- } \\
\text { sequence }\end{array}$} & \multicolumn{2}{|c|}{ Segment support } & \multirow{2}{*}{$\begin{array}{c}\text { Frequent 1- } \\
\text { sequence }\end{array}$} & \multicolumn{2}{|c}{ Segment support } \\
\cline { 2 - 3 } & Segment 1 & Segment 2 & & Segment 1 & Segment 2 \\
\hline$\langle\{\mathrm{A}\}\rangle$ & 2 & 2 & $\langle\{\mathrm{C}\}\rangle$ & 1 & 2 \\
$\langle\{\mathrm{~B}\}\rangle$ & 2 & 2 & $\langle\{\mathrm{D}\}\rangle$ & 1 & 1 \\
\hline
\end{tabular}

Lemma 1. If the maximum support of a sequence is less than the minimum support threshold, then the sequence is not a frequent sequence.

For example, the initial maximum support of $\langle\{\mathrm{A}\}\{\mathrm{B}\}\rangle$ is $2+2=4$, and the initial maximum support of $\langle\{A\}\{D\}>$ is $1+1=2$, which cannot be the frequent sequences.

Step 2. Generate $C_{k+1}$ from $C_{k}$, compute the initial maximum supports of the candidate $(k+1)$-sequences, and then prune candidate $(k+1)$-sequences which cannot be the frequent $(k+1)$-sequences from $C_{k+1}$.

For example, the initial maximum support of candidate 3 -sequence $\langle\{A\}\{B\}\{C\}>$ is $1+2=3$.

Step 3. Scan the transaction database $D B$ from the first segment to the last segment.

Lemma 2. If the maximum support of a sequence $s$ is equal to the current support of $s$, and the current support is no less than the minimum support, then $s$ is a frequent sequence.

Step 3.1. For the $i^{\text {th }}\left(i_{i} \grave{\mathrm{U}} 1\right)$ segment scan, SSLP records the position pairs of each candidate $k$-sequence corresponding to each customer sequence in the $i^{\text {th }}$ segment, computes the maximum support and the current support for each candidate $k$ sequence, and then prunes the candidate $k$-sequences which cannot be the frequent sequences. Besides, if the pruned candidate $k$-sequence is a subsequence of a candidate $(k+1)$-sequence $s$, then $s$ is also pruned from $C_{k+1}$.

For example, Table 3 shows the related information about some of $C_{2}$ after scanning the first segment, where candidate 2-sequence $\langle\{C\}\{A\}>$ can be pruned, because its maximum support is less than the minimum support. Besides, according to Lemma 
2, we can find some frequent sequences earlier, and these sequences need not be further examined.

Table 3. The related information for some candidate 2 -sequences and candidate 3 -sequences

\begin{tabular}{|c|c|c|c|c|}
\hline \multirow[t]{2}{*}{ Candidate } & \multicolumn{2}{|c|}{ Position pairs } & \multirow{2}{*}{$\begin{array}{l}\text { Maximum } \\
\text { support }\end{array}$} & \multirow{2}{*}{$\begin{array}{l}\text { Current } \\
\text { support }\end{array}$} \\
\hline & $\begin{array}{c}\text { Customer sequence } \\
1\end{array}$ & $\begin{array}{c}\text { Customer sequence } \\
2\end{array}$ & & \\
\hline $\begin{array}{c}<\{A\}\{B\}> \\
<\{A\}\{C\}> \\
<\{C\}\{A\}> \\
<\{A\}\{B\}\{C\}>\end{array}$ & $\begin{array}{c}(3,3) \\
(0,0) \\
(0,0) \\
0\end{array}$ & $\begin{array}{c}(2,3) \\
(3,3) \\
(0,0) \\
3\end{array}$ & $\begin{array}{l}4-(2-2)=4 \\
3-(1-1)=3 \\
3-(1-0)=2 \\
3-(1-1)=3\end{array}$ & $\begin{array}{l}2 \\
1 \\
0 \\
1\end{array}$ \\
\hline
\end{tabular}

Step 3.2. Find the $i$-segment support of each candidate $(k+1)$-sequence by computing the simple positions according to Definition 3, compute the maximum support and the current support for each candidate $(k+1)$-sequence, and then prune the candidate $(k+1)$-sequences which cannot be the frequent sequences according to Lemma 1.

Definition 3. Let a sequence $s=\left\langle a_{1}, \ldots, a_{k}\right\rangle$, and the two ( $k$-1)-subsequences of $s$ are $s_{1}=\left\langle a_{1}, \ldots, a_{k-2}, a_{k-1}\right\rangle$ and $s_{2}=\left\langle a_{1}, \ldots, a_{k-2}, a_{k}\right\rangle$. Assume that the position pairs of $s_{1}$ and $s_{2}$ corresponding to the customer sequence $c$ are $\left(f_{1}, l_{1}\right)$ and $\left(f_{2}, l_{2}\right)$, respectively. The simple position of s corresponding to the customer sequence $c$ is denoted as $\operatorname{Sim}_{s}(c)$. If $\mathrm{f}_{1}<\mathrm{l}_{2}$ and $\mathrm{f}_{1} ; \dot{U} 0, f_{2} ; U$ U then $\operatorname{Sim}_{s}(c)=1_{2}$ else $\operatorname{Sim}_{s}(c)=0$.

For example, the position pair of $\langle\{\mathrm{A}\}\{\mathrm{B}\}\rangle$ corresponding to customer sequence 4 is $(2,2)$, and the position pair of $\langle\{A\}\{C\}\rangle$ is $(2,3)$. Hence, the simple position of $<\{\mathrm{A}\}\{\mathrm{B}\}\{\mathrm{C}\}>$ corresponding to customer sequence is 3 .

Lemma 3. A customer sequence $c$ supports a sequence $s$ if and only if the simple position of $s$ corresponding to $c$ is not equal to 0.

According to Lemma 3, if the simple position of a candidate $(k+1)$-sequence $s$ corresponding to a customer sequence $\mathrm{c}$ is not equal to 0 , then $\mathrm{c}$ support $s$. Hence, the supports of all the candidate $(k+1)$-sequences can be found by computing the simple positions. Thus, we do not have to scan the transaction database $D B$ for counting the support of $s$.

SSLP repeats step 3.1 and step 3.2 until all the segments in the transaction database $D B$ are scanned, and the candidate sequences which are remained in $C_{k}$ and $C_{k+1}$ are the frequent sequences. In our example, SSLP finally generates $L_{2}$ and $L_{3}$ :

$L_{2}=\{\langle\{\mathrm{A}\}\{\mathrm{B}\}\rangle,\langle\{\mathrm{A}\}\{\mathrm{C}\}\rangle,\langle\{\mathrm{B}\}\{\mathrm{A}\}\rangle,\langle\{\mathrm{B}\}\{\mathrm{C}\}\rangle\}, L_{3}=\{\langle\{\mathrm{A}\}\{\mathrm{B}\}\{\mathrm{C}\}\rangle\}$. 


\section{Experimental Results}

We refer to [1] to generate three synthetic transaction databases, and evaluate the performance of SSLP by comparing this algorithm with Aprioriall.

Suppose the maximum length of the generated candidate sequences by Aprioriall is $q$. Hence, the maximum length of the generated frequent sequences is no greater than $q$. Aprioriall has to scan the given transaction database $q-1$ times, but SSLP needs only to scan the database $[q-1 / 2\rceil$ times. However, if $q$ is even, then the maximum length of the candidate sequences generated by SSLP may be $q+1$. If $C_{q+1}$ is not empty, SSLP need to compute the supports for the candidate $(q+1)$-sequences, even though there is no frequent $(q+1)$-sequences generated. The relative execution times for Aprioriall and SSLP over the minimum support ranging from $20.2 \%$ to $19 \%$, in which the segment length is 250 . The execution time of SSLP is between 1.6 times and 2.6 times as fast as that of Aprioriall. We notice that when the minimum support is $20 \%$, the performance gap slightly decreases because the maximum length of the candidate sequences generated by Aprioriall is even. SSLP outperforms Aprioriall ranging from 1.6 to 2.6 , and the performance gap increases as the minimum support decreases because the number of database scans increases for Aprioriall.

\section{References}

1. Agrawal R., et al.: Mining Sequential Patterns. Proceedings of International Conference on Data Engineering. (1995) 3-14

2. Agrawal R., et al.: Mining Sequential Patterns: Generalizations and Performance Improvements. Proceedings of the Fifth Int'l Conference on Extending Database Technology. Avignon, France (1996) 3-17

3. Agrawal R.: Johannes Gehrke, Dimitrios Gunopulos, Prabhakar Raghavan : Automatic Subspace Clustering of High Dimensional Data for Data Minig Applications. Proceedings of the ACM SIGMOD Int'l Conference on Management of Data. Seattle, Washington (1998) 94105

4. Agrawal R., Bayardo R. J., Srikant R.: Mining-based Interactive Management of Text Databases. IBM Research Report RJ10153. (1999)

5. Savasere A., Omiecinski E., Navathe S.: An Efficient Algorithm for Mining Association Rules in Large Databases. Proceedings of $21^{\text {st }}$ VLDB Conference. Zurich, Swizerland (1995) 432-444

6. Yen S.J., Chen A.L.P.: An Efficient Approach to Discovering Knowledge from Large Databases. Conference on Parallel and Distributed Information Systems. (1996) 8-18. 